\title{
Postoperative Etoricoxib versus Ketoprofen Administration for Pain Management after Total Knee Arthroplasty: A Randomized, Double-Blind Controlled Study
}

\author{
Simona Cionac Florescu, ${ }^{1}$ Denisa Madalina Anastase, ${ }^{1}$ \\ Ana Maria Munteanu, ${ }^{1}$ and Cristian Ioan Stoica ${ }^{2,3}$ \\ ${ }^{1}$ Department of Anesthesiology, Foisor Orthopedics Clinical Hospital, Bulevardul Ferdinand No. 35-37, 021382 Bucharest, Romania \\ ${ }^{2}$ Foisor Orthopedics Clinical Hospital, Bucharest, Romania \\ 3"Carol Davila" University of Medicine and Pharmacy, Strada Dionisie Lupu No. 37, 020022 Bucharest, Romania
}

Correspondence should be addressed to Denisa Madalina Anastase; danastase1@gmail.com

Received 30 August 2015; Revised 4 December 2015; Accepted 14 December 2015

Academic Editor: Thomas Engelhardt

Copyright ( 2015 Simona Cionac Florescu et al. This is an open access article distributed under the Creative Commons Attribution License, which permits unrestricted use, distribution, and reproduction in any medium, provided the original work is properly cited.

\begin{abstract}
Objective. This randomized double-blind study compared the analgesic efficacy and tolerability of etoricoxib versus ketoprofen in 165 patients with elective total primary knee arthroplasty. Methods. After ethical committee approval, 165 patients were randomized in 3 groups: the etoricoxib group (E) receiving etoricoxib $120 \mathrm{mg} / \mathrm{day}$, at the end of surgery and in the first postoperative day; the ketoprofen group $(\mathrm{K})$ receiving ketoprofen 2 pills of $100 \mathrm{mg} /$ day, the first at the end of surgery and then 1 pill every 12 hours in the surgery day and the first postoperative day; the placebo group (P). All groups received postoperatively the same analgesia protocol when NRS is over 3 with IV Perfalgan and morphine. The effectiveness was evaluated by the time from the initiation of spinal anesthesia until the first analgesic dose, the total amount of morphine administered in the surgery day and the first postoperative day, and the frequency of patients with side effects and necessary amount of adjuvant medication. Results. The baseline demographic characteristics were similar among the 3 groups. In both study days etoricoxib provided an analgesic effect superior to placebo and to ketoprofen, the total administered morphine being significantly lower in etoricoxib group. There were no statistically significant differences between groups regarding the side effects.
\end{abstract}

\section{Introduction}

In total knee replacement surgery, patients may have moderate to severe pain in the postoperative period. Because of side effects of opioids, postoperative analgesia with a combination of agents and techniques known as multimodal analgesia is recognized as the most effective practice in pain management [1].

The nonsteroidal anti-inflammatory drugs (NSAIDs) provide good pain relief when combined with opioids in multimodal analgesia regimens, but guidelines generally recommend patients to discontinue them due to the inhibition of cyclooxygenase-1 (COX-1) by traditional NSAIDs, which has been shown to interfere with platelet aggregation leading to the possibility of increased bleeding in surgical wound [2].
Ketoprofen is a NSAID derivative of propionic acid. Although used as a racemic mixture, the pharmacodynamics activity and its anti-inflammatory effect is due entirely to the $S$ $(+)$ enantiomer (dexketoprofen), while the $\mathrm{R}(-)$ enantiomer is devoid of such activity [3]. It is very highly bound to plasma protein, reaches maximum plasma concentration occuring after 6-8 hours, and declines thereafter with a half-life of about 8 hours; also it diffuses into the synovial fluid. It is metabolized primarily by conjugation and eliminated mainly by the kidneys [4].

The appearance of coxibs, selective inhibitors of COX2 agents, brought advantages in postoperative pain management because they are not associated with the side effects of NSAIDs and therefore can be used more safely [5].

A representative of this class is etoricoxib (Arcoxia) which has demonstrated efficacy in many painful conditions. It has 
the largest selectivity of the coxibs (COX-2/COX-1 of 106) [6], a good absorption rate, and the half-life of 22 hours allowing administration of a single $120 \mathrm{mg}$ pill per day. Literature data show that the installation of its action is just 24 minutes after the administration [7].

The aim of this prospective double-blinded randomized study was to investigate the effect of etoricoxib versus ketoprofen in reducing the amount of morphine required for postoperative analgesia following total knee replacement. In this setting total morphine consumption, duration of anesthesia, the side effects of drugs administered, and the necessary amount of adjuvant medication were evaluated.

\section{Materials and Methods}

After the approval of local ethics committee and signed informed consent of the patients, 165 ASA I-II patients with age between 20 and 85 years, weight over $40 \mathrm{~kg}$, height above $155 \mathrm{~cm}$, and Hct $\geq 30 \%$, with primary degenerative joint disease undergoing elective total primary knee replacement were included in the study; the surgery technique was the same in all patients.

Patients with history of bronchial asthma, endocrine disorders, severe hepatic and renal diseases, neuropathies, bleeding disorders, preexisting gastric ulcers, gastritis and history of gastrointestinal bleeding, dementia, cooperation disability, and sensitivity to the drugs used in study were not included. Also patients with long-acting NSAID administered in the last four days before surgery were not included in the study, like those with cerebrovascular or peripheral arterial disease, congestive heart failure (NYHA II-IV), or HTA not controlled appropriately $[4,8]$.

The patients who agreed to consent were enrolled and randomized by a computer generated random number list (GraphPad Prism 6, http://www.graphpad.com/) prepared by an investigator with no clinical involvement in the trial. The result of the randomization (the allocation for groups $\mathrm{E}, \mathrm{K}$, or $\mathrm{P})$ was written on a note placed inside a numbered envelope (one number corresponding to every patient enrolled). The main investigator was the only person who had access to the randomization code and who stored it. Another investigator not involved in patient care and unaware of the allocation group of the patient opened the sealed envelope and performed the administration of the pills.

Patients were randomised in 3 groups of 55 patients each:

(1) The etoricoxib group (E) received etoricoxib 1 pill of $120 \mathrm{mg}$ orally postoperatively at the end of surgery and a second etoricoxib $120 \mathrm{mg}$ pill after 24 hours (at the beginning of the first postoperative day); this group also received 1 sham pill every 12 hours intercalated between the two active doses of etoricoxib.

(2) The ketoprofen group $(\mathrm{K})$ received ketoprofen 1 pill of $100 \mathrm{mg}$ orally at the end of surgery and then 1 pill every 12 hours during the surgery day and first postoperative day.

(3) The patients in the placebo group received sham pills in the same way (at the end of surgery and every $12 \mathrm{~h}$, during the surgery day and first postoperative day). We used placebo pills with the same appearance as ketoprofen.

To maintain the blinding conditions, medical staff had no knowledge of the randomization of the patients or the contents of the pills.

The pills were administered at the end of the surgery, immediately after the patients arrived in the PACU, and no one had nausea and vomiting.

2.1. Anesthetic Technique. All patients had $0.01 \mathrm{mg} / \mathrm{kg}$ intravenous midazolam for premedication (Dormicum, Roche, Switzerland). The operations were performed under spinal anesthesia with $0.5 \%$ isobaric bupivacaine using 25 - or 27 gauge Whitacre needles. Propofol $2-3 \mathrm{mg} / \mathrm{kg} / \mathrm{hour}$ was given for the intraoperative sedation.

2.2. Postoperative Analgesia. Postoperatively all subjects were transferred in the postoperative care unit (PACU) during the study period. Pain was assessed by the duty nurses at the first postoperative demand for analgesia by the patients either verbally or by pushing a button. Evaluation of postoperative pain intensity was performed using the numerical rating scale (NRS, from 0 to 10 ), the patient being informed about it preoperatively. We defined as "duration of analgesia" the time from spinal puncture to the first analgesic demand.

The multimodal analgesia plan was started when patients began to experience pain (NRS > 3) by the administration of paracetamol (Perfalgan, Bristol-Myers Squibb, New York, USA) in a fixed and scheduled dose $1 \mathrm{~g}$ IV infusion for 15 minutes every 8 hours. If after paracetamol infusion NRS remains over 3, morphine (morphine hydrochloride, Sanofi Aventis Zentiva, Paris, France) was administered according to the IV titration protocol approved and applied in PACU of our hospital (Figure 1). This analgesia protocol was also applied in the first postoperative day.

Simultaneously with the first dose of opioid injection ondansetron (Osetron, Dr. Reddy's, India) was administered at a dose of $4 \mathrm{mg}$ IV.

Adverse events such as sedation, nausea, vomiting, dryness of mouth, sweating, urinary retention, respiratory depression, hypotension, tachycardia, and surgery bleeding were recorded during the study.

Sedation was evaluated by a four-point scale: (1) awake, (2) reacting to the verbal stimulant, (3) reacting to the painful stimulant, and (4) no reaction to the painful stimulant.

The respiratory rate below 8 per minute was defined as a respiratory depression and treated with repeated doses of naloxone to remission.

Heart rate below 50 per minute was considered as bradycardia and treated with IV atropine; also hypotension was considered as the lowering of mean arterial pressure by more than $30 \%$ of its preoperative value and IV ephedrine was administered.

In case of pruritus a loratadine (Claritin) tablet was indicated. 

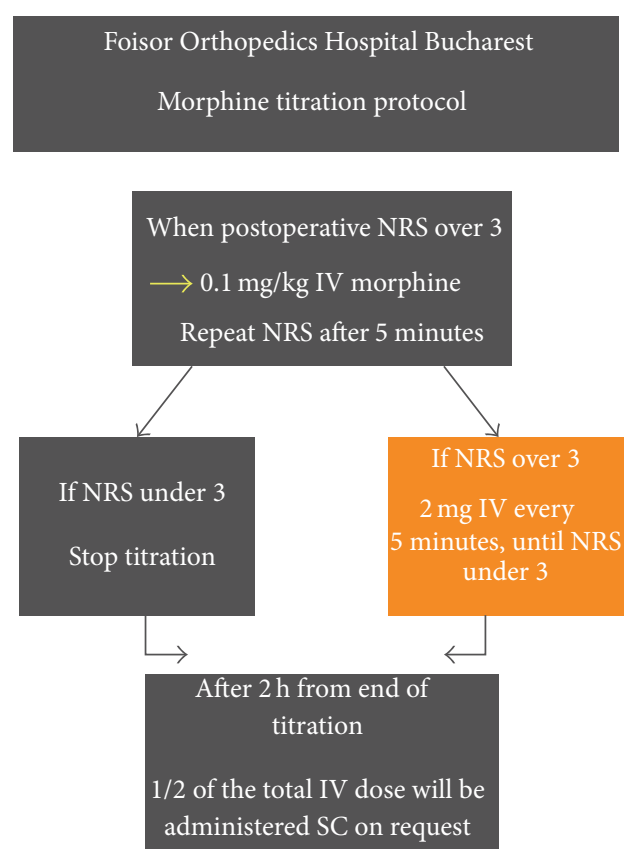

FIGURE 1: Postoperative morphine titration protocol (Foisor Hospital, Bucharest).

During the study we monitored the following parameters:

(i) The amount of morphine required during the first 24 hours (day of surgery) and next 24 hours (first postoperative day), at rest as the primary outcome.

(ii) The duration of analgesia defined as the time from the initiation of spinal anesthesia until the patient asked for the first dose of analgesic as the secondary outcome.

(iii) The frequency of patients with side effects, the necessary amount of adjuvant medication also as the secondary outcome.

2.3. Statistical Analysis. We used GraphPad StatMate 2 and GraphPad Prism 6 (http://www.graphpad.com/). The calculation of the sample size relied on the primary outcome (postoperative morphine consumption), which from our previous studies was $40 \pm 14 \mathrm{mg}$ SD in the placebo group [9]. In order to demonstrate a difference of the means (delta) of 5-7 points between the two drugs, not between drugs and placebo only, with a power of $85 \%$ and a type I error (alfa) of 0.05 , we found that each group should have at least 50 patients. We analyzed the data based on intention-to-treat principle.

Either chi-square or Fisher's exact test was used to analyze categorical outcomes. Differences among 3 groups of continuous variables were analyzed by analysis of variance (ANOVA). Post hoc analysis was performed with Tukey test.

\section{Results}

3.1. Demographic Data. Data are presented as mean \pm standard deviation $(x \pm \mathrm{SD})$. The results are considered statistically significant if the $p<0.05$.
TABLE 1: Demographic data.

\begin{tabular}{lccc}
\hline Group & Etoricoxib & Ketoprofen & Placebo \\
\hline Number & 55 & 55 & 55 \\
Age, yrs & $66.2 \pm 8.6$ & $70.1 \pm 6.4$ & $65.0 \pm 7.7$ \\
Weight, kg & $76.8 \pm 14$ & $79 \pm 12.5$ & $70.1 \pm 11$ \\
Height, cm & $162.7 \pm 7.6$ & $163.6 \pm 16$ & $163.3 \pm 7.5$ \\
Sex, f/m & $41 / 14$ & $44 / 11$ & $46 / 9$ \\
\hline
\end{tabular}

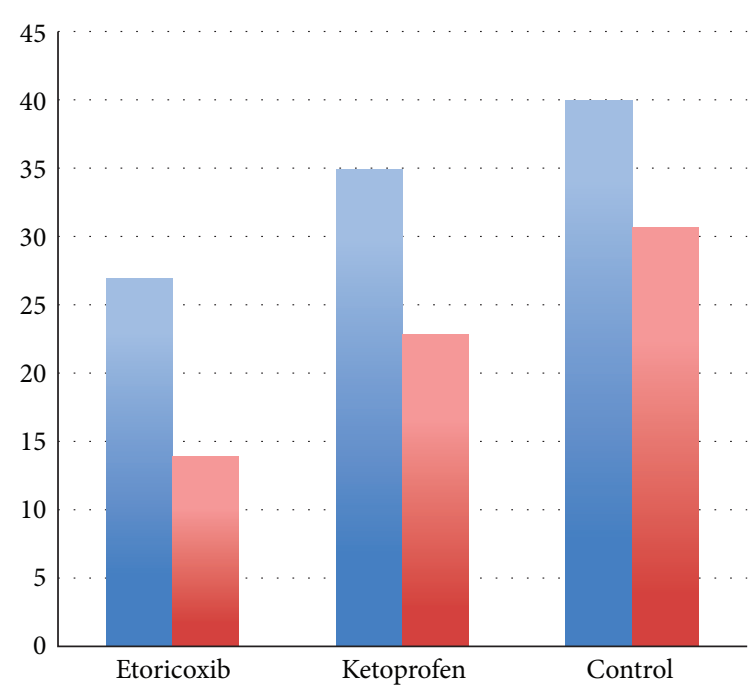

$$
\begin{aligned}
& \text { Surgery day } \\
& \text { Postoperative day } 1
\end{aligned}
$$

FIGURE 2: Morphine consumption (mg).

The three groups were similar in terms of age, weight, height, gender, American Society of Anesthesiologists (ASA) class, number of patients, and surgery duration (Table 1).

3.2. Morphine Consumption. The average morphine consumption on the day of surgery was $27 \mathrm{mg}$ for etoricoxib group, $35.02 \mathrm{mg}$ for ketoprofen group, and $40.15 \mathrm{mg}$ for placebo group (Table 2, Figure 2). On the first postoperative day the means were $13.58 \mathrm{mg}$ for group E, $22.73 \mathrm{mg}$ for group $\mathrm{K}$, and $30.95 \mathrm{mg}$ for group $\mathrm{P}$ (Table 2, Figure 2).

One-way ANOVA followed by Tukey's multiple comparisons test were performed. The results showed a significant effect of Etoricoxib compared to placebo $(p<0.0001)$ and to ketoprofen $(p<0.004)$ to reduce morphine requirements in the first $24 \mathrm{~h}$ postoperative and in the first postoperative day (Table 3).

3.3. The Duration of Analgesia. The average duration of analgesia was 337 minutes for group E, 283.45 minutes for group K, and 266.36 minutes for group P (Table 4, Figure 3 ). Tukey's multiple comparisons test confirmed a significant analgesic effect of Etoricoxib compared to placebo and to ketoprofen (Table 5). For better understanding the type of distribution we used D'Agostino \& Pearson normality test and 
TABLE 2: Morphine consumption (mg).

\begin{tabular}{|c|c|c|c|c|c|c|c|}
\hline \multirow{2}{*}{ Group } & \multirow{2}{*}{ Mean (mg) } & \multirow{2}{*}{ Std. deviation } & \multirow{2}{*}{ Std. error } & \multicolumn{2}{|c|}{ 95\% confidence interval for mean } & \multirow{2}{*}{ Minimum } & \multirow{2}{*}{ Maximum } \\
\hline & & & & Lower bound & Upper bound & & \\
\hline \multicolumn{8}{|c|}{ Morphine consumption on the day of surgery } \\
\hline Group E & 27.00 & 14.26 & 1.92 & 30.63 & 38.34 & 7.50 & 72.00 \\
\hline Group K & 35.02 & 13.85 & 1.87 & 31.27 & 38.76 & 12.00 & 88.50 \\
\hline Group P & 40.15 & 11.76 & 1.59 & 36.97 & 43.33 & 15.00 & 88.00 \\
\hline \multicolumn{8}{|c|}{ Morphine consumption on postoperative day 1} \\
\hline Group E & 13.58 & 9.14 & 1.23 & 11.11 & 16.05 & 0.0 & 34.5 \\
\hline Group K & 22.73 & 11.28 & 1.51 & 19.69 & 25.76 & 6.0 & 59.5 \\
\hline Group P & 30.94 & 13.16 & 1.77 & 27.40 & 34.51 & 11.0 & 92.0 \\
\hline
\end{tabular}

TABLE 3: Morphine consumption-results of multiple comparisons Tukey's test.

\begin{tabular}{|c|c|c|c|c|c|}
\hline Tukey's multiple comparisons test & Mean diff. & 95\% CI of diff. & Significant & Summary & $p$ \\
\hline \multicolumn{6}{|c|}{ Morphine consumption on the day of surgery } \\
\hline Etoricoxib versus ketoprofen & -7.8 & -14 to -2.1 & Yes & $* *$ & 0.0044 \\
\hline Etoricoxib versus placebo & -13 & -19 to -7.2 & Yes & $* * * *$ & $<0.0001$ \\
\hline Placebo versus ketoprofen & 5.1 & -0.85 to 11 & No & Ns & 0.1090 \\
\hline \multicolumn{6}{|c|}{ Morphine consumption on the postoperative day 1} \\
\hline Etoricoxib versus ketoprofen & -9.8 & -15 to -4.3 & Yes & $* * * *$ & $<0.0001$ \\
\hline Etoricoxib versus placebo & -18 & -23 to -13 & Yes & $* * * *$ & $<0.0001$ \\
\hline Placebo versus ketoprofen & 8.2 & 3.1 to 13 & Yes & $* * *$ & $<0.0006$ \\
\hline
\end{tabular}

TABLE 4: Duration of analgesia (min).

\begin{tabular}{lcccccccc}
\hline Group & \multirow{2}{*}{ Mean } & Std. dev. & \multicolumn{2}{c}{ 95\% confidence interval for mean } & Median & 25\% percentile & 75\% percentile & Normality test passed \\
\hline Group E & 337 & 89 & 295 & 344 & 320 & 275 & 380 & No \\
Group K & 283 & 76 & 262 & 304 & 285 & 240 & 330 & Yes \\
Group P & 247 & 48 & 236 & 256 & 245 & 210 & 285 & Yes \\
\hline
\end{tabular}

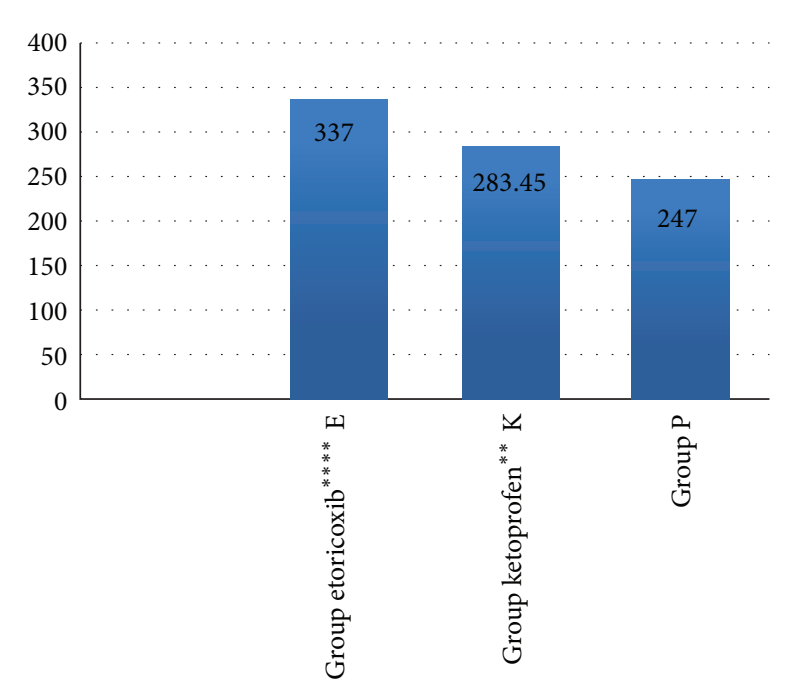

Figure 3: Mean duration of analgesia (min).

calculated also median and interquartile range of duration of analgesia (minutes).
3.4. The Side Effects. The overall incidence of adverse events in the 3 groups of the study was small. There were no statistically significant differences between groups regarding the side effects.

The most common side effects of morphine administration were nausea, vomiting, bradycardia, and sedation; there were no significant differences between groups (Table 6).

There were no reported specific side effects related to etoricoxib administered in the 2 study days.

\section{Discussion}

The total knee arthroplasty is one of the best orthopedic interventions that reduces painful symptoms and improves the functionality and mobility of patients with osteoarthritis, increasing the quality of life [10].

But these operations can be associated with significant postoperative pain. In addition to patient discomfort and satisfaction, inadequate pain management determines many medical complications contributing to a prolongation of the length of hospital stay, readmission to hospital, and higher costs [11]. 
TABLE 5: Duration of analgesia-results of multiple comparisons Tukey's test.

\begin{tabular}{|c|c|c|c|c|c|}
\hline Tukey's multiple comparisons test & Mean diff. & 95\% CI of diff. & Significant & Summary & $p$ \\
\hline Etoricoxib versus ketoprofen & 51 & 21 to 82 & Yes & $* * *$ & $<0.0003$ \\
\hline Etoricoxib versus placebo & 90 & 59 to 121 & Yes & $* * * *$ & $<0.0001$ \\
\hline Placebo versus ketoprofen & -39 & -69 to -7.8 & Yes & $* *$ & $<0.0097$ \\
\hline
\end{tabular}

TABLE 6: The side effects.

\begin{tabular}{lccc}
\hline \multirow{2}{*}{ Side effects } & \multicolumn{3}{c}{ Number of cases/group } \\
& Group E & Group K & Group P \\
\hline Vomiting & 3 & 4 & 6 \\
Nausea & 3 & 4 & 4 \\
Sedation & 2 & 3 & 3 \\
Bradycardia & 1 & 1 & 1 \\
Dizziness & 0 & 1 & 1 \\
O2 desaturation & 0 & 0 & 1 \\
\hline
\end{tabular}

In the treatment of postoperative pain, opioids are used as first-line drugs, but because of their side effects they are combined with NSAIDs. Analgesic action of NSAIDs is carried out by inhibition of inducible COX-2 in peripheral tissues, nerves, and the central nervous system; however, by blocking also constitutive COX-1 they cause gastrointestinal toxicity and affect platelet function, increasing the risk of bleeding, which limits their use perioperatively [2].

The COX-2 selective inhibitors spare the use of opioids without affecting platelet function, finding an important place in the postoperative pain management of patients [12].

Several clinical trials evaluated the efficacy and safety of etoricoxib in reducing postoperative pain in orthopedic surgery compared with placebo or active comparator, either preemptively or postoperatively [13-15].

Thus, Rasmussen et al. showed that etoricoxib $120 \mathrm{mg}$ after surgery in patients with TKA or THA provided analgesia superior to placebo and similar to naproxen $1100 \mathrm{mg}$ [16]. The results of a study of Rawal et al. showed that etoricoxib $120 \mathrm{mg}$ was administered also postoperatively in patients with TKA effects superior to placebo and equal to ibuprofen $1800 \mathrm{mg}$ to reduce pain at rest and opioid consumption [17]. These results are overlapping investigations by Lin et al. recently published in 2013, who showed the benefits of perioperative administration of COX-2 inhibitors [18].

Studies that compare NSAIDs with coxibs to treat postoperative pain are rare $[2,19]$.

In our double-blind, placebo controlled, randomized clinical study, we evaluated postoperative pain following knee replacement in patients receiving placebo, etoricoxib $120 \mathrm{mg}$, or ketoprofen $200 \mathrm{mg}$ daily.

Etoricoxib had a rapid onset of pain relief and prolonged duration of action in comparison to placebo and ketoprofen in the first 24 hours postoperatively, which is consistent with data published in the literature concerning the treatment of postoperative pain in major orthopedic surgery [20].

The benefits of etoricoxib became more evident in the first postoperative day, with an important reduction of rescue medication use, the total amount of morphine being significantly less than in other groups of patients.

Another important variable is the duration of analgesia, which is the highest in etoricoxib group providing an increased degree of patient satisfaction.

Etoricoxib was well tolerated during the 2-day study; the incidence of adverse events was infrequent and similar across treatment groups.

According to the European Medicines Agency (EMEA) when coxibs are prescribed in accordance with contraindications and precautions, the balance between the risks versus benefit remains positive in target patients [21].

Some limitations of our study refer to the fact that we have assessed pain only at rest and did not follow up the evolution of patients in terms of cardiovascular complications longer than hospital stay (mean 6.3 days).

\section{Conclusions}

In this clinical study, in the treatment of pain following a total knee arthroplasty, etoricoxib at a dose of $120 \mathrm{mg}$, administered postoperatively and in the first postoperative day, demonstrated a greater pain control as compared with placebo and ketoprofen $200 \mathrm{mg}$ daily, evidenced by a significant decrease in opioid use, with a greater duration of anesthesia.

\section{Conflict of Interests}

The authors declare that there is no conflict of interests regarding the publication of this paper.

\section{References}

[1] A. Buvanendran and J. S. Kroin, "Multimodal analgesia for controlling acute postoperative pain," Current Opinion in Anaesthesiology, vol. 22, no. 5, pp. 588-593, 2009.

[2] P. F. White, J. Tang, R. H. Wender et al., "The effects of oral ibuprofen and celecoxib in preventing pain, improving recovery outcomes and patient satisfaction after ambulatory surgery," Anesthesia and Analgesia, vol. 112, no. 2, pp. 323-329, 2011.

[3] N. Sivrikoz, K. Koltka, E. Güresti, M. Büget, M. Sentürk, and S. Özyalçın, "Perioperative dexketoprofen or lornoxicam administration for pain management after major orthopedic surgery: a randomized, controlled study," Agri, vol. 26, no. 1, pp. 23-28, 2014.

[4] http://www.drugs.com/pro/ketoprofen.html.

[5] R. Sinatra, "Role of COX-2 inhibitors in the evolution of acute pain management," Journal of Pain and Symptom Management, vol. 24, no. 1, supplement 1, pp. S18-S27, 2002. 
[6] D. Riendeau, M. D. Percival, C. Brideau et al., "Etoricoxib (MK0663): preclinical profile and comparison with other agents that selectively inhibit cyclooxygenase-2," Journal of Pharmacology and Experimental Therapeutics, vol. 296, no. 2, pp. 558-566, 2001.

[7] J. K. Takemoto, J. K. Reynolds, C. M. Remsberg, K. R. Vega-Villa, and N. M. Davies, "Clinical pharmacokinetic and pharmacodynamic profile of etoricoxib," Clinical Pharmacokinetics, vol. 47, no. 11, pp. 703-720, 2008.

[8] http://ec.europa.eu/health/documents/community-register/ html/ho2001.htm.

[9] D. Anastase, S. Florescu-Cionac, A. M. Munteanu et al., "Is there any analgesic benefit from preoperative vs. postoperative administration of etoricoxib in total knee arthroplasty?" Regional Anesthesia and Pain Medicine, vol. 39, no. 5, supplement 1, 2014.

[10] M. S. Ibrahim, M. A. Khan, I. Nizam, and F. S. Haddad, "Perioperative interventions producing better functional outcomes and enhanced recovery following total hip and knee arthroplasty: an evidence-based review," BMC Medicine, vol. 11, article 37, 2013.

[11] J. Parvizi and M. R. Bloomfield, "Multimodal pain management in orthopedics: implications for joint arthroplasty surgery," Orthopedics, vol. 36, no. 2, pp. 7-14, 2013.

[12] S. S. Reuben, R. Fingeroth, R. Krushell, and H. Maciolek, "Retracted: evaluation of the safety and efficacy of the perioperative administration of rofecoxib for total knee arthroplasty," The Journal of Arthroplasty, vol. 17, no. 1, pp. 26-31, 2002.

[13] A Randomized Controlled Trial to Study the Efficacy of Pre-emptive Etoricoxib in Reducing Postoperative Pain and Improving Functional Outcome in Total Knee Arthroplasty, Identifier: NCT01246362, Clinical Trials Overview December 2012, https://clinicaltrials.gov/.

[14] ClinicalTrials.gov, "A phase III randomized, double-blind, placebo- and active-comparator-controlled, multiple-dose, clinical trial to study the safety and efficacy of MK0663/etoricoxib and ibuprofen in the treatment of postorthopedic knee replacement surgery pain," Clinical Trials Overview, 2013, https://clinicaltrials .gov/ct2/show/NCT00820027.

[15] J. Fleckenstein, S. Kramer, M. Offenbächer et al., "Etoricoxibpreemptive and postoperative analgesia (EPPA) in patients with laparotomy or thoracotomy-design and protocols," Trials, vol. 11, article 66, 2010.

[16] G. L. Rasmussen, K. Malmstrom, M. H. Bourne et al., "Etoricoxib provides analgesic efficacy to patients after knee or hip replacement surgery: a randomized, double-blind, placebocontrolled study," Anesthesia \& Analgesia, vol. 101, no. 4, pp. 1104-1111, 2005.

[17] N. Rawal, E. Viscusi, P. M. Peloso et al., "Evaluation of etoricoxib in patients undergoing total knee replacement surgery in a double-blind, randomized controlled trial," BMC Musculoskeletal Disorders, vol. 14, article 300, 2013.

[18] J. Lin, L. Zhang, and H. Yang, "Perioperative administration of selective cyclooxygenase-2 inhibitors for postoperative pain management in patients after total knee arthroplasty," Journal of Arthroplasty, vol. 28, no. 2, pp. 207-213, 2013.

[19] L. Wickerts, M. Warrén Stomberg, M. Brattwall, and J. Jakobsson, "Coxibs: is there a benefit when compared to traditional non-selective NSAIDs in postoperative pain management?" Minerva Anestesiologica, vol. 77, no. 11, pp. 1084-1098, 2011.

[20] R. Clarke, S. Derry, and R. A. Moore, "Single dose oral etoricoxib for acute postoperative pain in adults," Cochrane
Database of Systematic Reviews, vol. 5, Article ID CD004309, 2014.

[21] European Medicines Agency concludes action on COX-2 inhibitors, 2005, http://www.ema.europa.eu/. 


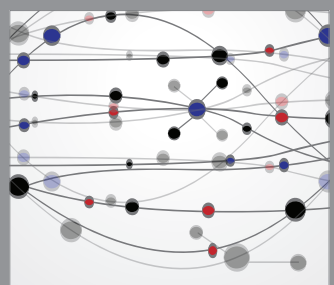

The Scientific World Journal
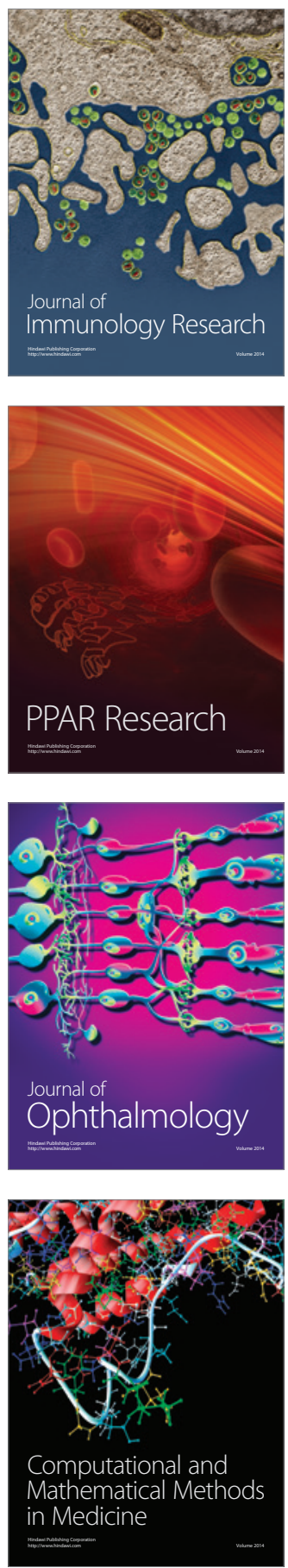

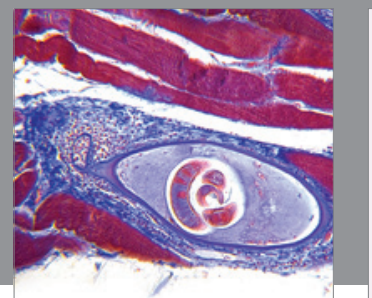

Gastroenterology

Research and Practice
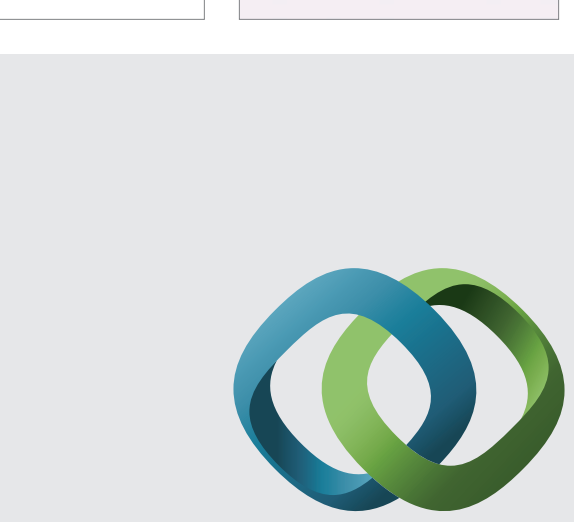

\section{Hindawi}

Submit your manuscripts at

http://www.hindawi.com
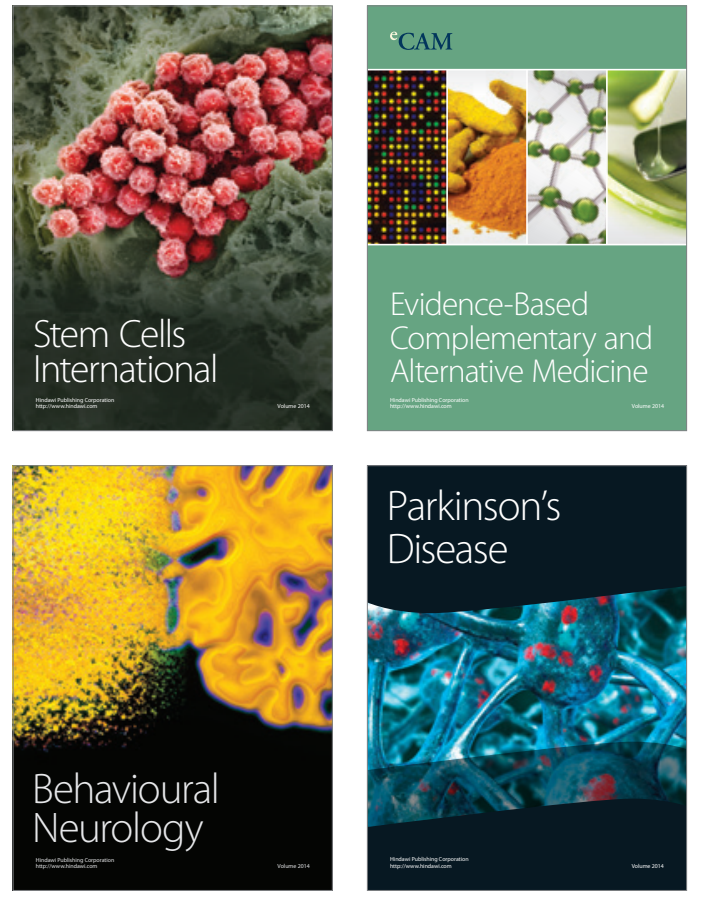
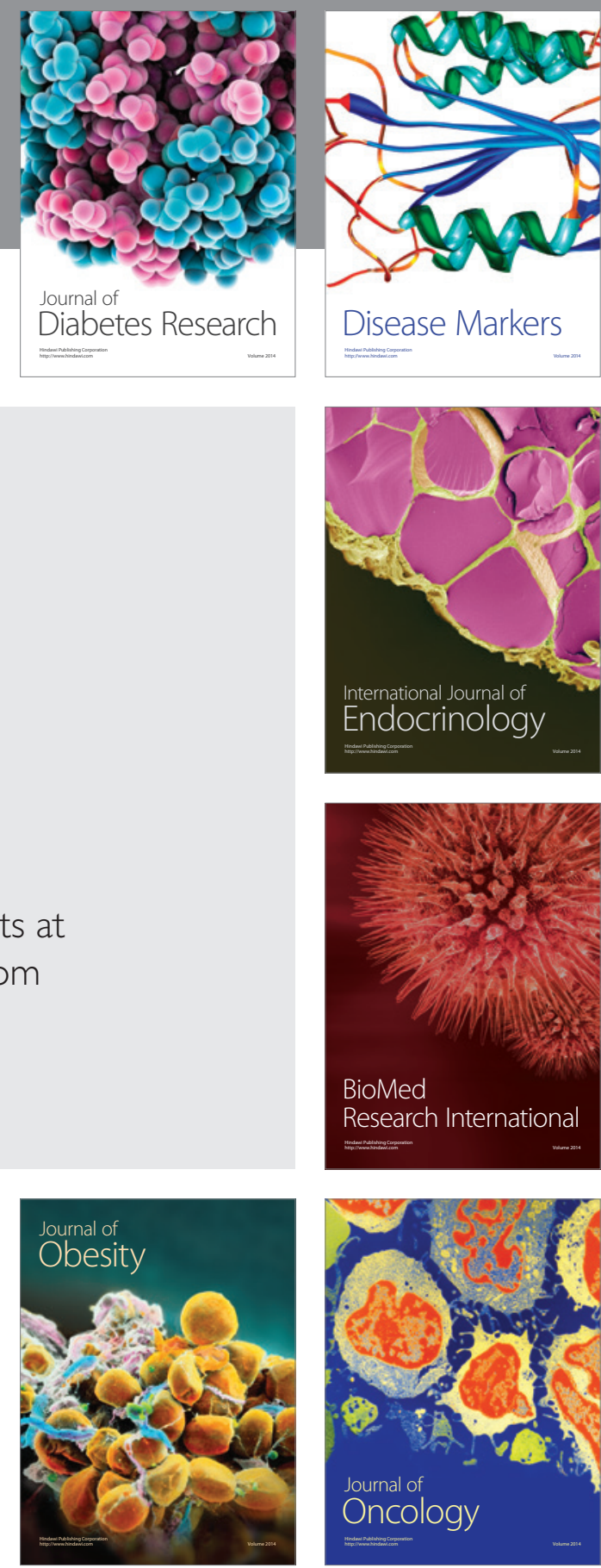

Disease Markers
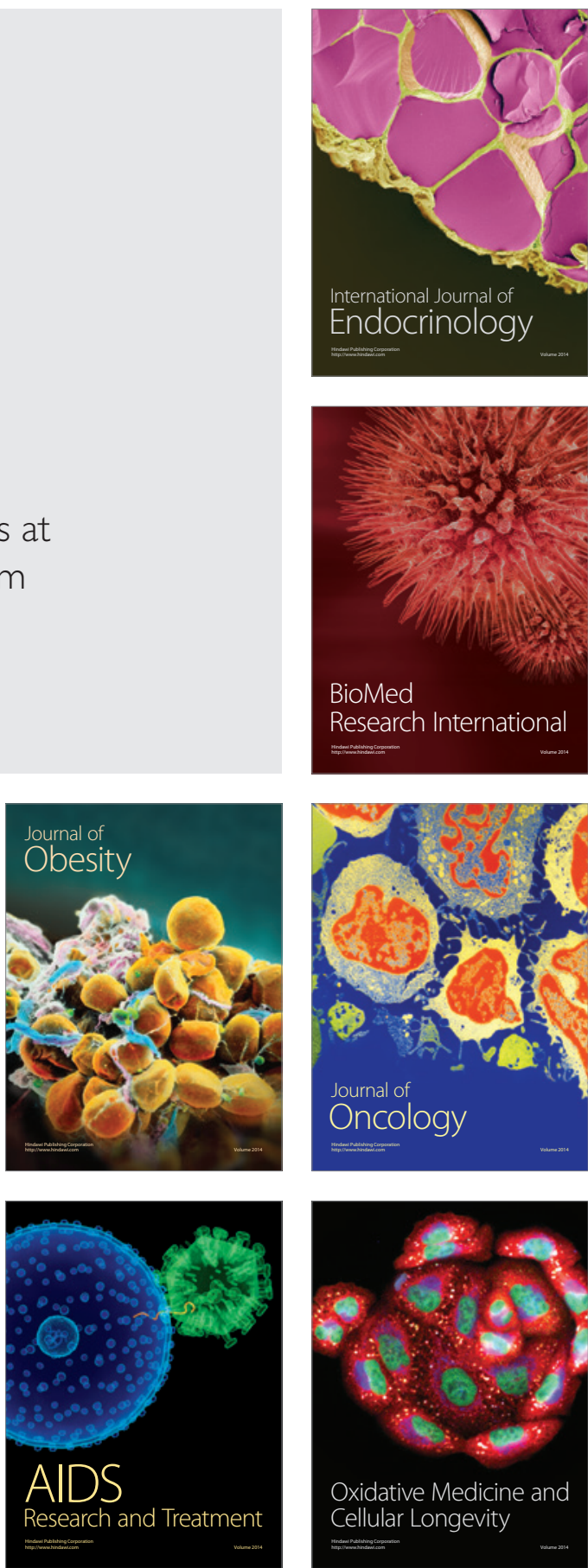